\title{
Measuring Sound Pressure Levels during Thermoacoustic Instabilities in Large Engines: Case Study
}

\author{
Alice Elizabeth González, José Cataldo Ottieri, Pablo Gianoli Kovar, \\ Joaquín Montero Croucciée, Marcos Raúl Lisboa
}

Department of Environmental Engineering, IMFIA-Faculty of Engineering, Universidad de la República, Montevideo, Uruguay Email: aliceelizabethgonzalez@gmail.com, elizabet@fing.edu.uy

How to cite this paper: González, A.E., Ottieri, J.C., Kovar, P.G., Croucciée, J.M. and Lisboa, M.R. (2017) Measuring Sound Pressure Levels during Thermoacoustic Instabilities in Large Engines: Case Study. Journal of Modern Physics, 8, 1685-1699. https://doi.org/10.4236/jmp.2017.810099

Received: July 19, 2017

Accepted: August 31, 2017

Published: September 4, 2017

Copyright $\odot 2017$ by authors and Scientific Research Publishing Inc. This work is licensed under the Creative Commons Attribution International License (CC BY 4.0).

http://creativecommons.org/licenses/by/4.0/

\begin{abstract}
This paper attempts to present some registers of sound pressure levels during the operation of large diesel engines (10 MW). During these registers we have found the preparation, occurrence and ending of events of thermoacoustic instability. They appear after a loosing of chaos period or a reduction in fluctuations in some frequencies. The most interesting phenomena were registered at low frequencies. However, they were accompanied by variations in sound emissions at medium and high frequencies. As there has been very little published data concerning these phenomena at real scale, it is imperative to point out that every quasi-stationary state we have measured during these episodes has lasted some minutes, significantly much more time than that of lab scale results.
\end{abstract}

\section{Keywords}

Thermoacoustic Instabilities, Large Engines, Real Scale Measurements

\section{Introduction}

Thermoacoustic instabilities are a growing concern when working with large machines. A lot of work has been developed about gas turbines, but very few experiences are reported measuring this phenomenon in real scales engines.

Noise in diesel engines is highly consistent with the variation of the pressure inside each cylinder along the time. As combustion has random features, the cylinder pressure and the emitted noise are dominated by randomness over a wide range of frequencies where noise is produced [1].

When working for determining the acoustic power of large engines (10 MW), 
we detected some unusual patterns in several third octave bands (TOB) recordings. We went through the analysis and we found we have measured some thermoacoustic instabilities episodes during the tests.

Thermoacoustic oscillations cause increased damage on the engines. The high sound pressure levels associated with the oscillations that occur during thermoacoustic instability, impose an additional load on the wall of the combustion chamber. In addition, non-stationary flow increases heat transfer to the coating and local overheating may occur. Even the electronic systems that control combustion could fail due to high levels of vibration or temperature, leading loss of the system control.

According to Schemel et al. (2004) when the flow is homogeneous, the noise in a combustion chamber is an overlapping of the solution of three independent wave equations: the waves traveling upflow and downflow at sound speed, the turbulent convective waves and the entropy convective waves. If the flow ceases to be homogeneous, the entropy convective waves will begin to emit (radiate) sound [2].

Combustion instabilities arise due to complex feedback interactions between pressure and heat release oscillations. When these oscillations are sufficiently in phase, a large amplification of the initial perturbation is expected. Thus, the instabilities of the combustion refer to the feedback of a coherent phase oscillation at a fixed frequency.

The pressure oscillations occurring in the combustion chamber appear as fluctuations in the output flow of the injector. As a consequence, fluctuations in the incoming air flow occur in turn. Then, fluctuations in the release of heat would also appear. The frequency of these oscillations depends on the main cause of them. Low frequency oscillations (from $4 \mathrm{~Hz}$ to $70 \mathrm{~Hz}$ ) are mainly due to instabilities in the flame front progressing in a heterogeneous way throughout the combustion chamber. They are related to low frequency emissions. From 70 $\mathrm{Hz}$ to $700 \mathrm{~Hz}$ stationary waves with different phase angles appear [3]. If those oscillations couple with the operating frequencies of the equipment, high intensity noise emissions will be released.

Polifke et al. (2001) point out that the feedback between the combustion chamber acoustics and the entropy waves would be important, especially for lower modes and even at higher frequencies than those normally associated with convection waves [4]. The relative phase between the acoustic signal at the combustion chamber outlet and the pressure pulse generated by the entropy wave determines whether the combustion chamber's susceptibility to thermoacoustic oscillations is improved or reduced by the interaction between the entropy waves and the acoustics of the combustion chamber.

The main issues affecting the occurrence of interferences are fluctuations and heterogeneities in fuel concentration, temperature regions, rate of heat release and also localized phenomena at the inlet and the fuel injection point. Depending on the characteristic times of the convection and acoustic phenomena, the 
entropy waves and the acoustic of the combustion chamber could couple in a constructive or destructive way.

It should be noted that the rotational frequencies of the machines in our case of study are within the proper range for flame front fluctuations.

The most important mechanisms responsible for instability in combustion are relatedtothe injection of propellants, the formation of liquid droplets and the combustion process itself. If a high temperature region passes through a supersonic nozzle (i.e. where $\mathrm{Ma}>1$ ), the interaction with the non-uniform flow region developed produces an acoustic wave which propagates upstream. Then, the action of the acoustic wave in the combustion processes can generate new regions of non-uniform temperature, with the usual consequences on the stability of the combustion. Therefore, there is a feedback loop within the chamber which maintains the oscillatory phenomena and which, under certain conditions, may lead to a condition of instability. The evolution of eddies can excite oscillations, either by purely fluid interactions or by influencing combustion processes [5]. The first one leads to relatively weak instabilities because the available mechanical energy is relatively small. In contrast, when combustion is involved, significant oscillations can occur. Eddies can carry reagents and due to delays or chemicals or in the mixing time, the combustion may subsequently occur at times and in spatial locations favorable to destabilize an acoustic mode.

The oscillations in pressure and velocity in the gas phase (acoustic disturbances) can influence the rate of vaporization of the liquid droplets if the period of oscillation corresponds to one of the characteristic times of the vaporization, namely [6]: life time of the drop; period of thermal inertia of the liquid; period of thermal diffusion of the liquid; period of diffusion of the gas phase of the gas mixture contained in the combustion chamber; period of diffusion of the gas phase by forced convection.

According to Schuermans (2003), fluctuations in fuel concentration are the main (but not the only) cause of the interaction between the heat release and the sound field [7]. Litak et al. (2005) conclude that the noise level of the internal pressure when calculating the entropy of the variations of the maximum pressures in successive cycles is not monotonous function of the load [3]. The results show that the combustion dynamics is a non-linear, multidimensional process mediated by noise. This method allows distinguishing a particular signal, including chaos with its short-term prediction scale, and random noise.

The "soul" of the problem is that all the ideal processes under which combustion is studied in small machines are no longer valid in large ones. The hypotheses about instantaneous ignition, homogeneity of the mixture and all phenomena occurring inside of each cylinder are no longer applicable.

This paper is organized in four sections. After this introduction, some ways to early detecting thermoacoustic instabilities are presented. Then, our own experimental findings when measuring at several $10 \mathrm{MW}$ enginesare detailed. At last, our conclusions are remarked. 


\section{Early Detection of Thermoacoustic Instabilities}

Research about thermoacoustic instabilities has mostly developed around gas turbines and at a laboratory scale. There are not enough published data about real scale cases. There are many proposals for detection of the occurrence of these phenomena. Hereby we present three of them; we think our experimental findings can help to deeper studies on them.

\subsection{Loss of Chaos}

The phenomenon of loss of chaos was studied by Vinneeth et al. (2013) [8]. They present convincing evidence that combustion noise is deterministic. Therefore, the traditional "signal plus noise" paradigm that is usually implicitly assumed in the models and analyzes of experimental data sets needs to be re-examined if capturing instabilities in the combustion chambers is wanted because these irregular fluctuations might contain useful prognostic information. Vinneeth et al. generate a way to predict the impending passage to unstable combustion by applying the 0 - 1 test for chaos on sequentially acquired pressure measurements. For low level of combustion noise the measured $\mathrm{K}$ value is quite close to 1 in the initial stages, indicating that the combustion noise is chaotic. The value of $\mathrm{K}$ has a decreasing tendency as the Reynolds number of the flux increases, reaching values close to 0 at the beginning of the instability. Since the loss of the chaos condition occurs in a gentle manner, the value of $\mathrm{K}$ can be used as a measure of the proximity of an imminent instability condition. Choosing a threshold value of $\mathrm{K}$ that corresponds to the initial stages of loss of chaos (e.g. 0.9), it is possible to know the condition enough in advance to take actions that modify the operation parameters and thus avoid the occurrence of instability and therefore its subsequent installation as an operating regime [8].

The precursor turns out to be an objective measure of the proximity of the combustion chamber to the unstable operating regimes and is independent of the details of the geometry, the composition of the fuel and the stabilization of the flame.

\subsection{Rapid Detection by Analyzing Trends of Variation}

During the preparation of a thermoacoustic instability event, some early "symptoms" should be detected, thus allowing taking actions to avoid the instability occurrence. Ibrahim (2007) proposes the use of a low-cost method which implies having a good identification and characterization of several acoustic modes to be able to follow its temporal evolution and to know about its growing and decreasing tendencies. This background allows to make a good prediction without numerically integrating over time: its detection tool analyzes the behavior of rates of variation and not modes, which is undoubtedly simpler and faster. The method consists in cataloging and linearly estimating of magnitudes of the mechanisms of amplification and attenuation. The application of linear approximations to nonlinear mechanisms allows, however, obtaining a reasonably com- 
plete and manageable description for the purposes of the analysis. The author defines an index of stability, so that the imminent occurrence of an instability is anticipated when a certain value is exceeded. In this particular case, the oscillation is expected to occur when the value of the index exceeds one unit [9].

The goal of the method is to allow fast, low-cost decisions that can be made for a wide variety of design configurations and operating conditions without the complexity of other tools that require computational fluid dynamics. The proposed approach achieves moderate success by being tested on a basis of experimental data available in the literature as well as with new experiments, so it may also be useful to complement other methods already in use.

\subsection{Experimental Diagnosis}

Lee and Santavicca (2005) carry out an extensive discussion of the applicable methods for experimentally diagnosing, i.e. in operation, if instability is occurring in a combustion chamber [10]. Among the methods they discuss, brief comments are made here on some of them. Pressure measurements are usually the simplest ones, although it is necessary to take a set of precautions, for example about the location of the sensor or how to fix it, in order to obtain information in accordance with the objective. In addition to measuring pressure fluctuations in the combustion chamber, it is useful to simultaneously measure pressure fluctuations in the nozzle and in the fuel line. These fluctuations result in fluctuations in the rate of fuel flow, a phenomenon known as coupling of the feed system. Such measurements provide valuable information for evaluating the coupling role of the feed system in terms of its role as a mechanism in conducting or damping/aborting the effective occurrence of instability [10].

The effect of entropy waves on flow field instabilities is known since 1965, but their importance was supposed to be restricted only to low frequencies. More recent works (e.g., [4]) show that interference between entropy waves and pressure disturbances can be constructive or destructive, which can further aggravate the problem.

Fluctuations of heat release in the flame can cause acoustic waves that propagate upstream in the feed lines and in turn cause disturbances in the incoming air/fuel mixture. These disturbances can be carried by the mean flow and trigger a fluctuation in the flame controller, closing the instability loop. Several studies have addressed this possible mechanism and are considered of high potential to generate instability phenomena. The acoustic-convective waves are carried by the medium flow, such as eddies detached from the flame stabilizer and/or entropy waves that propagate downstream, and generate acoustic waves that propagate upstream.

There are also other possible sources of oscillatory combustion instability ranging from purely chemical-kinetic phenomena to other only fluid-mechanical phenomena. Their contributions vary with modes of oscillation. It is also possible that some of the modes of oscillation are caused by a combination of 
perturbations (velocity, temperature, velocity of laminar flame, etc.).

Several mechanisms contribute to the occurrence of thermoacoustic instabilities [11]: fluctuations in the air/fuel ratio, acoustic-convective waves and entropy waves. Eddies detachment was suggested as the cause of combustion instabilities in 1956 by Rogers and Marble [12]. Instability is triggered when the vortices that are released from the flame stabilizer, entrain unburned mixture that spreads downstream and cause a sudden release of heat at some point in its path. This triggers an acoustic wave propagating upstream that closes the feedback loop.

A similar result would be found if these vortices affect an obstacle downstream (e.g. the outlet nozzle, a throttling, etc.), even if they carry no unburned mixture or if it is a non-reactive flow or a cold flow, causing the pressure oscillations to intensify. This result is purely acoustic and does not consider the contributions related to heat releasing.

Since hot spots are carried by the medium (usually low-speed) flow, it is assumed that entropy effects (if exist) are to occur at low frequencies. When these hot spots reach the entrance of a strangulated nozzle, the propagation of an upflow acoustic wave is triggered and it can cause an acoustic instability.

\section{Experimental Findings}

\subsection{Measurements}

A set of measurements were carried out to determine the acoustic power of eight large diesel engines (10 MW each). They were done according to UNE-EN-ISO 3744:2010 Standard [13]. A Class 1 Bruel \& Kjaer sound pressure level meter (Model 2250) was used. Also the environmental sound pressure levels in the engines room were recorded during the tests.

All the figures in this section have been built using the experimental data registered during the tests; please notice that all graphics relate to only one enginein operation.

\subsection{High Frequencies' Findings}

We found three kinds of phenomena that are to be called as cases A, B and C.

Case A refers to some high frequency components that became coherent from some time during the test (first presented at Figure 1 and Figure 2).

Case $\mathrm{B}$ is related to some simultaneous jumps occurring also at high frequencies (first presented at Figure 3).

Case $\mathrm{C}$ show reduced variability and increasing sound pressure levels in some high frequency TOB (initially presented at Figure 4 and Figure 5). They are preceded or accompanied by episodes of loss of chaos at low frequencies, mainly at $25 \mathrm{~Hz}$.

The first case (Case A) shows a qualitative change at the highest frequency waves at about 11:15: they become coherent as shown by the pattern they exhibit from $10,000 \mathrm{~Hz}$ and upper frequencies.It doesn't happen at lower frequencies (Figure 1 and Figure 2). 


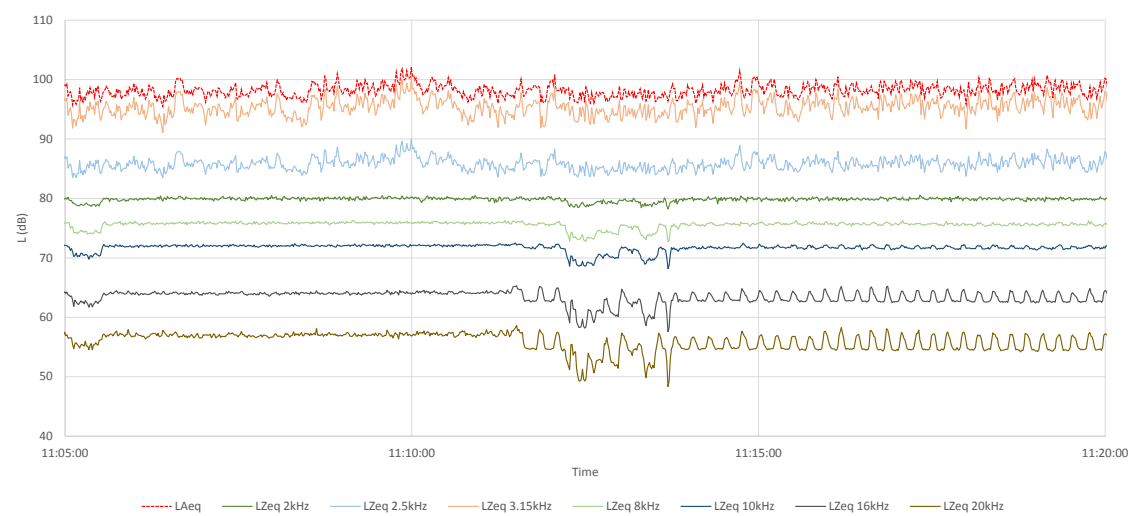

Figure 1. Time evolution of sound pressure levels in selected third-octave bands (Case A). Please note the change of shape occurring near 11:15 at the graphs of the highest frequencies.

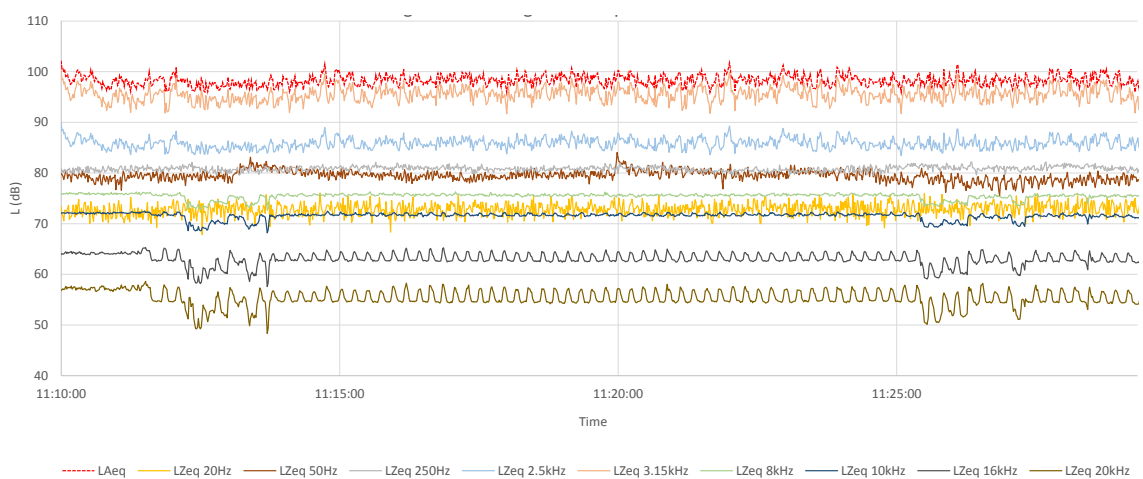

Figure 2. Loss of chaos at the highest audible frequencies (Case A).

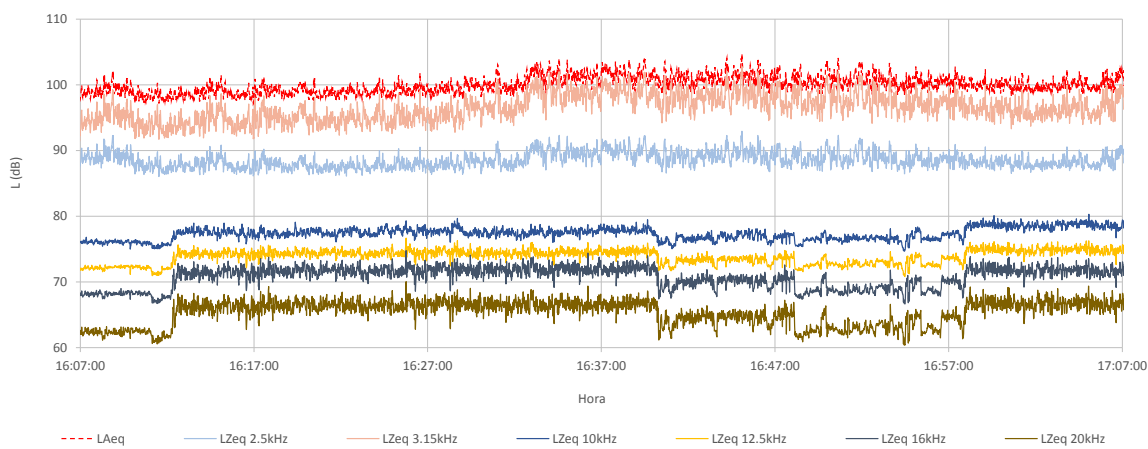

Figure 3. Time evolution of sound pressure levels in selected third-octave bands (Case B). Please note the simultaneous jumps occurring at the highest frequencies.

Another kind of phenomena appears at high frequencies in Case B. No coherence phenomena appear but there are some ascending and descending jumps that occur within some 3 to 5 minutes of difference. Although they are not reflected in the broad band levels, sound pressure levels jumps occur simultaneously in several TOBs (Figure 3).

The third case we found at high frequencies (Case C) is shown in Figure 4 and Figure 5. The sound pressure levels at high frequencies reduce their varia- 


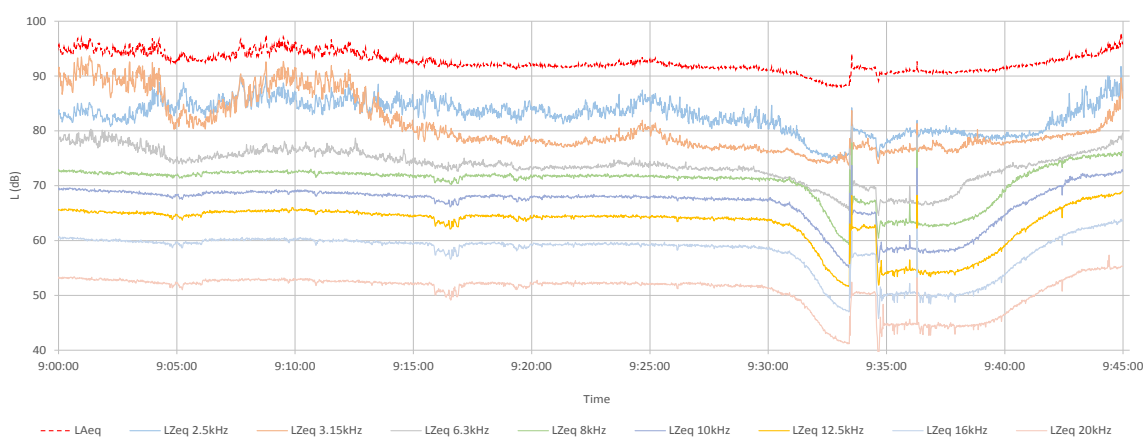

Figure 4. Time evolution of sound pressure levels in selected third-octave bands (Case C). Please note the decreasing variability of sound pressure levels at the highest audible frequencies.

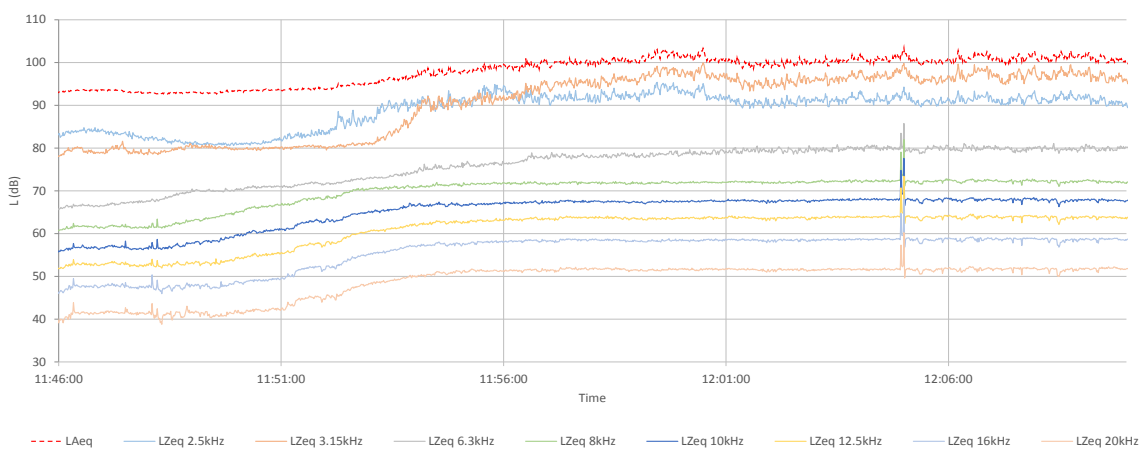

Figure 5. Time evolution of sound pressure levels in selected third-octave bands (Case C). Decreasing variability of sound pressure levels with increasing levels at the highest audible frequencies.

bility while increasing their value.

This occurs from $2500 \mathrm{~Hz}$ and upper frequencies. The sound pressure levels at $2000 \mathrm{~Hz}$ are rather constant over the time and have very few fluctuations.

We have also registered interference phenomena during noise measures, as shown in Figure 6. Graphs show destructive interferences whether the components in different TOB appear almost in opposition of phase. It was found to happen both at harmonic frequencies (left) and at non-harmonic ones (right).

\subsection{Related Phenomena at Low Frequencies: The Main Causes}

Once we have identified what was happening at high frequencies, we went on looking for regularities at other frequencies. We found that the root causes in the above-mentioned cases were linked to changes at low frequencies.

The changes in regime of acoustic emissions in TOB of $25 \mathrm{~Hz}$ but also $12.5 \mathrm{~Hz}$ and $50 \mathrm{~Hz}$ were always present during the recorded events.

We had only one measurement where Case A occurred (coherence in high frequencies components). For this particular case, we found that the component in $50 \mathrm{~Hz}$ was qualitatively less chaotic in the previous 10 minutes (Figure 7) and that was a consequence of an extended process which began about 50 minutes earlier (Figure 8). 


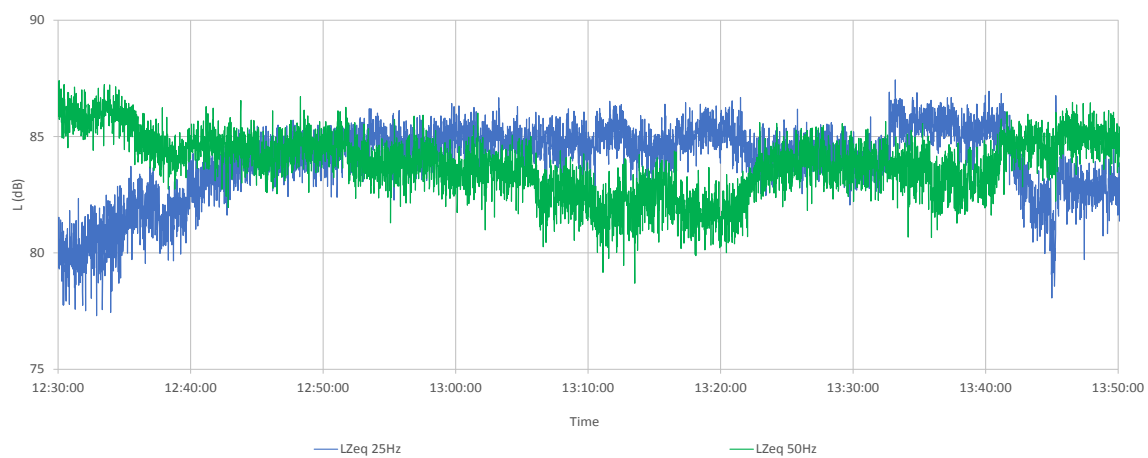

(a)

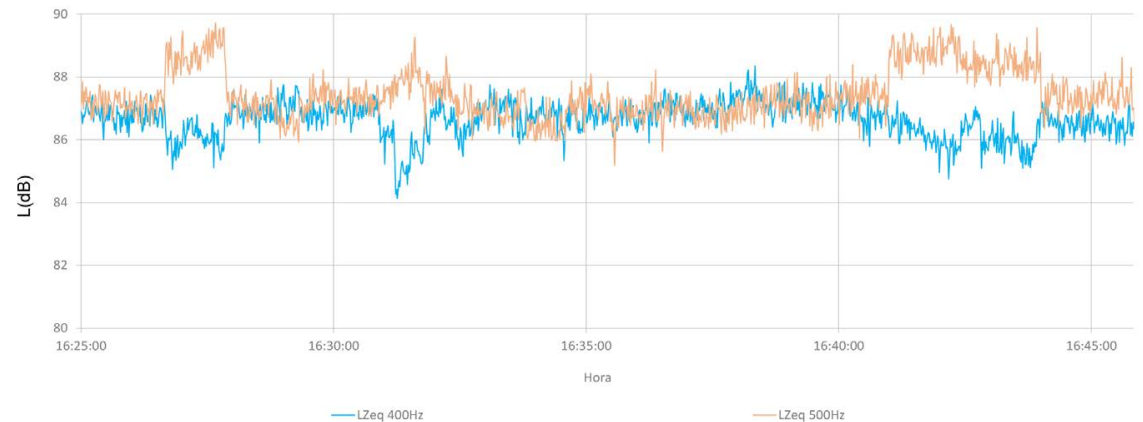

(b)

Figure 6. Destructive interferences in selected third-octave bands. (a): low frequencies 25 $\mathrm{Hz}$ and $50 \mathrm{~Hz}$; (b): middle frequencies $400 \mathrm{~Hz}$ and $500 \mathrm{~Hz}$.

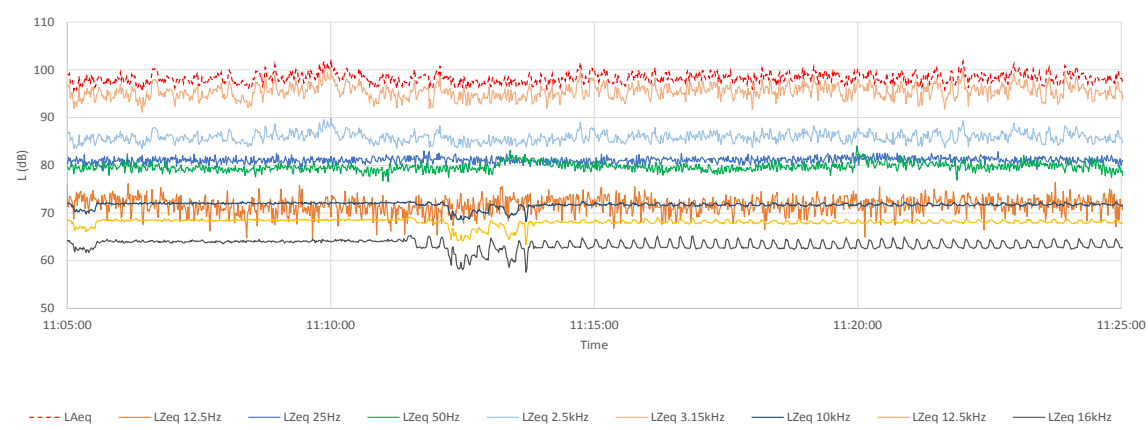

Figure 7. Previous changes in $16 \mathrm{~Hz}$ and $50 \mathrm{~Hz}$ about 10 minutes earlier than the beginning of Case A.

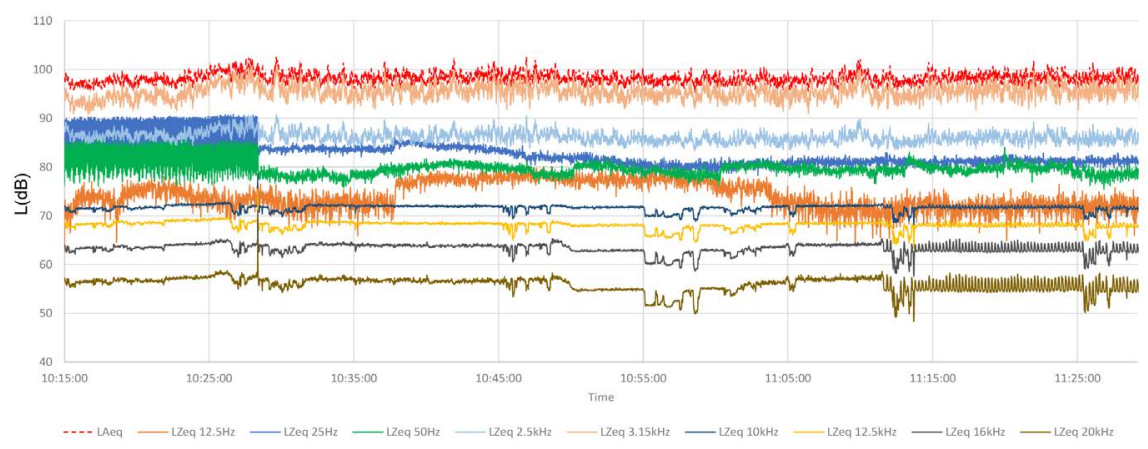

Figure 8. Different steps during Case A preparation: loss of coherence at $25 \mathrm{~Hz}$ (1 hour earlier) and sequence of different quasi-stationary states (about 10 minutes each one). 
Case B was the most frequent during our measurements. It can be seen as a set of jumps in sound pressure levels occurring simultaneously in several TOB. There are at least three of these jumps in Figure 9. It corresponds to the whole event presented in Figure 3. It is possible to observe that jumps in high frequencies are anticipated by changes at lower frequencies expressed as episodes of loss of chaos.

Figures 10-13 show the same kind of phenomena occurring in other events.

The third group of cases we measured (Cases C) had the most unexpected behaviour at low frequencies.

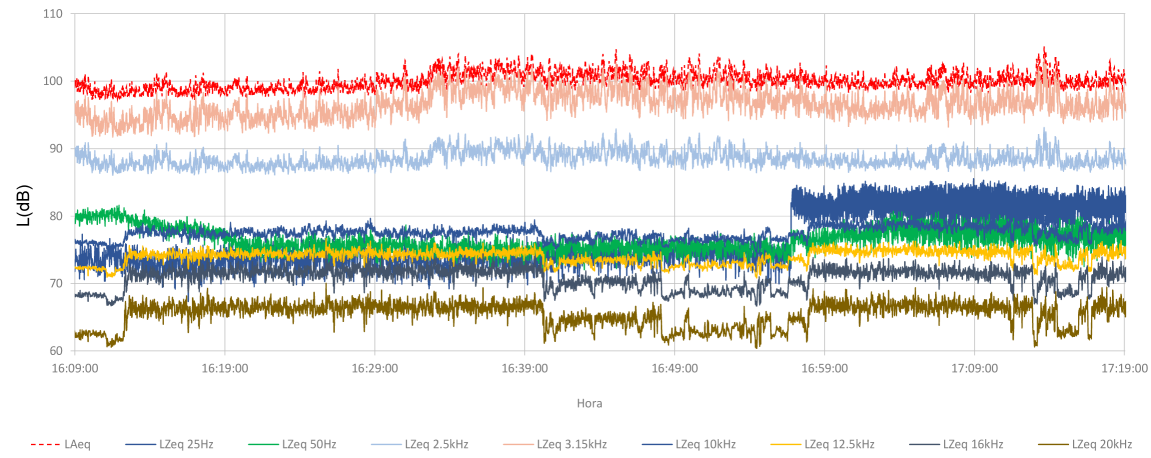

Figure 9. Simultaneous jumps in sound pressure levels at different TOB (Case B).

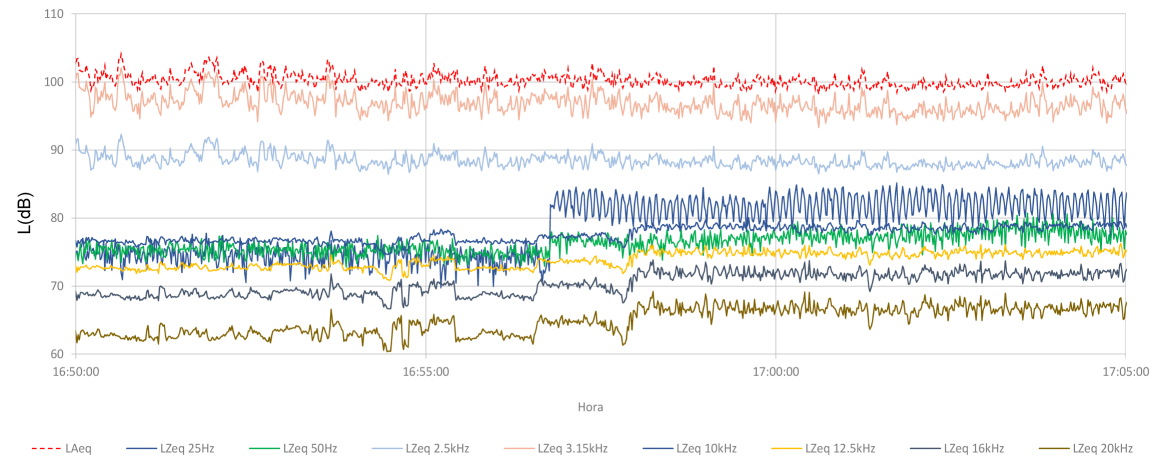

Figure 10. Measured sound pressure levels in selected TOB. Detail of Case B presented in Figures 3-9. Please note the great change at $25 \mathrm{~Hz}$ TBO.

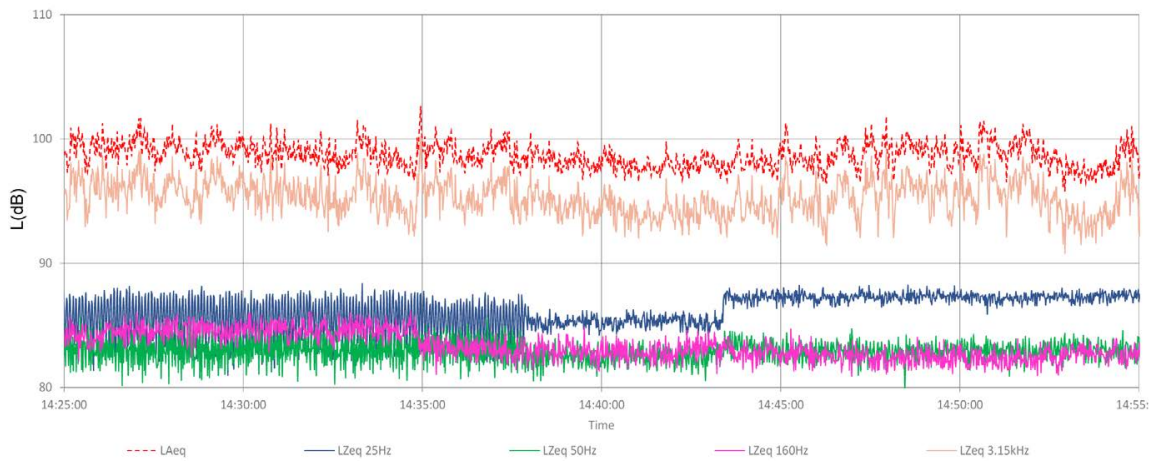

Figure 11. Examples of occurrence of Case B. Please note jumps at $25 \mathrm{~Hz}, 50 \mathrm{~Hz}$ and 160 $\mathrm{Hz}$. 
Figure 14 shows the evolution of some TOB during the whole event and Figure 15 presents a detail of what happens at TOB of $25 \mathrm{~Hz}$ and $50 \mathrm{~Hz}$. When sound pressure levels and variability at $2.500 \mathrm{~Hz}$ begin to increase, low frequency bands return to a more chaotic condition. Note that sound pressure levels at certain middle TOB (e.g. $160 \mathrm{~Hz}$ and $315 \mathrm{~Hz}$ ) show similar behavior than highest frequencies.

Another interesting example is shown in Figure 16 and Figure 17. The increase of sound pressure levels at high frequencies (e.g. $2500 \mathrm{~Hz}$ and $3150 \mathrm{~Hz}$ ) is

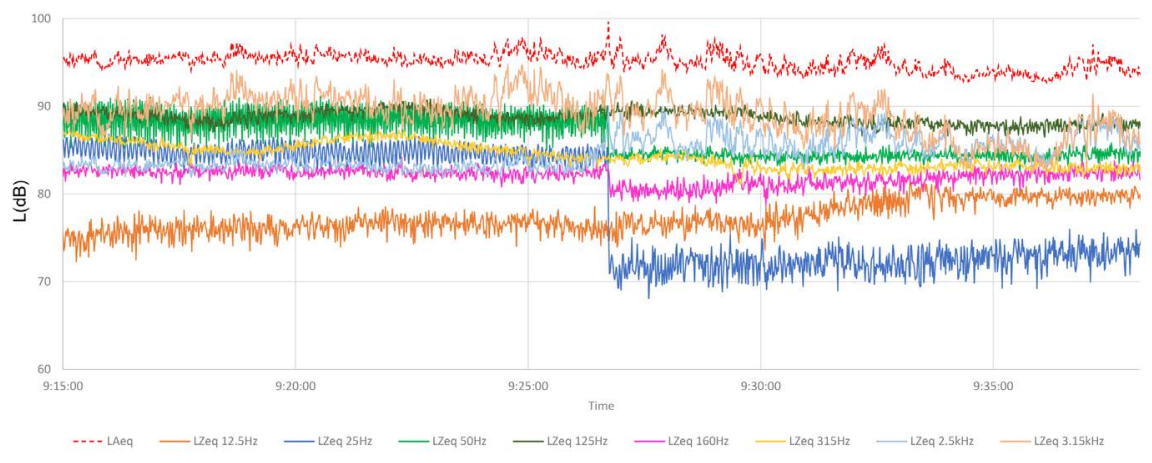

Figure 12. Examples of occurrence of Case B. Jumps at $25 \mathrm{~Hz}, 50 \mathrm{~Hz}$ and $160 \mathrm{~Hz}$ are accompanied by smooth changes at $12.5 \mathrm{~Hz}, 125 \mathrm{~Hz}$ and $315 \mathrm{~Hz}$.

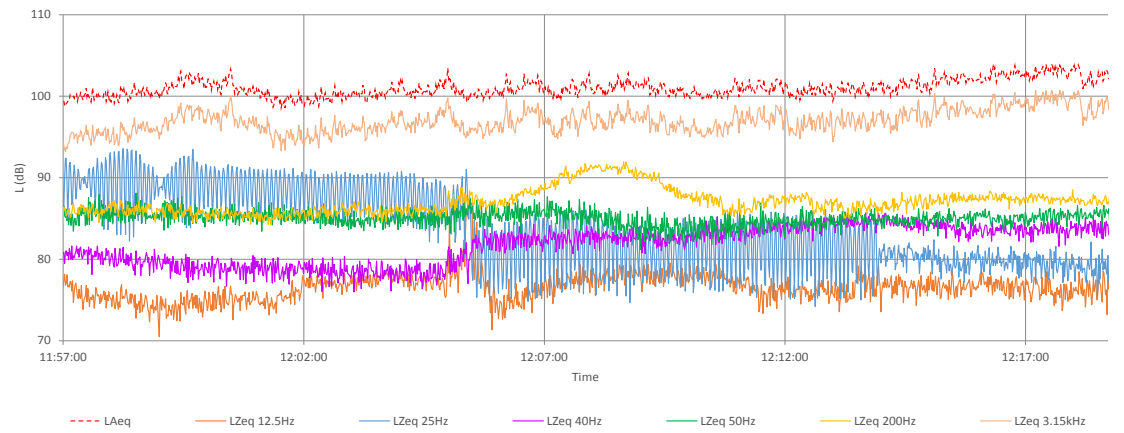

Figure 13. Examples of occurrence of Case B. Changes of behaviour at $25 \mathrm{~Hz}$ are accompanied by others at several TOB e.g. $12.5 \mathrm{~Hz}, 40 \mathrm{~Hz}$ and $200 \mathrm{~Hz}$.

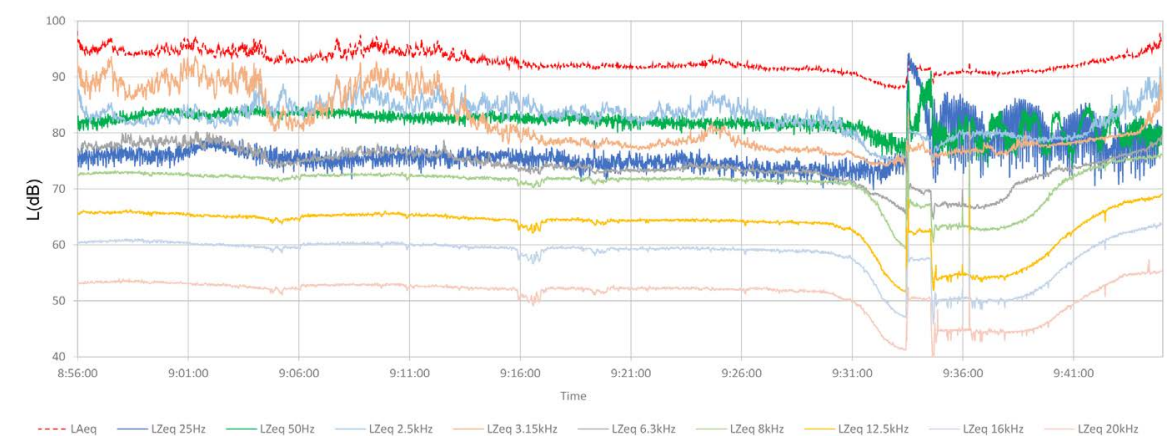

Figure 14. Example of occurrence of Case C. The phenomena at $25 \mathrm{~Hz}$ and $50 \mathrm{~Hz}$ occur after loss of variability and reduction of sound pressure levels at high frequencies; then, they begin to grow again. 
very smooth; it lasts more than one hour since the beginning of the phenomenon at low frequencies $(25 \mathrm{~Hz}$ and $50 \mathrm{~Hz})$, as it can be seen in a closer approach shown at Figure 17.

Finally, Figure 18 and Figure 19 show the evolution of some TOB during the whole event introduced in Figure 5. Figure 20 presents a closer approach of the retrieve of chaos at low frequencies. Two different scales seem to be involved in the phenomena: a quasi-periodic wave and a modulating one. This behaviour seems to appear in Case B, but in a less evident way.

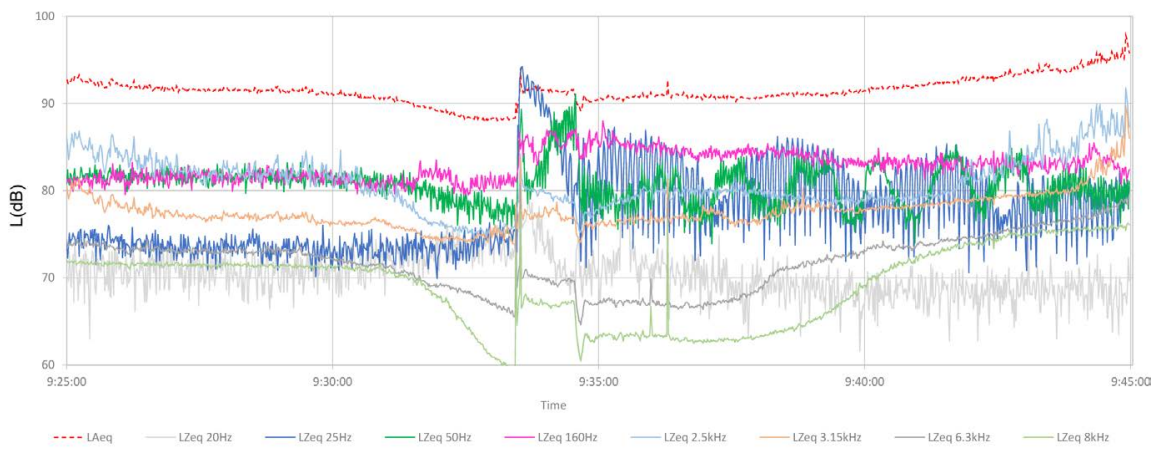

Figure 15. Measured sound pressure levels in selected TOB. Detail of Case C presented in Figure 14.

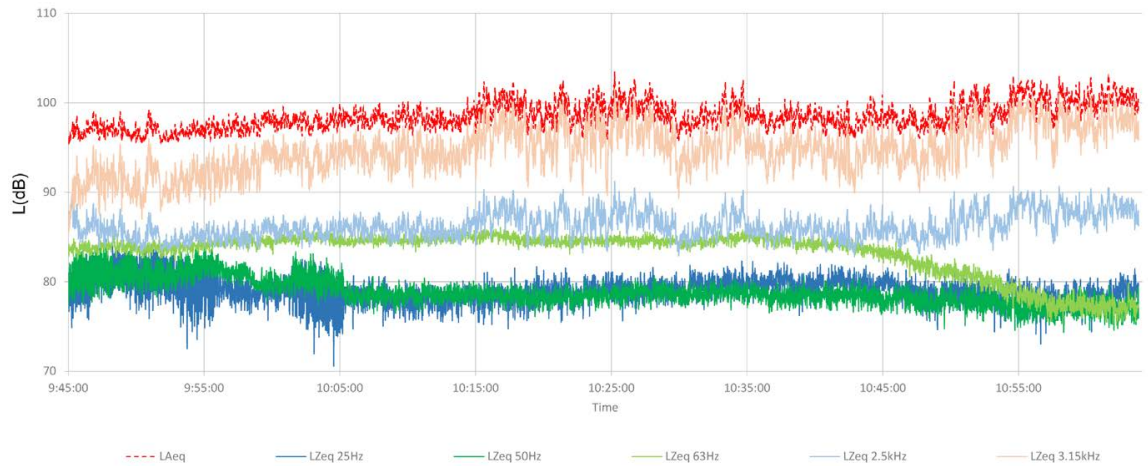

Figure 16. Examples of occurrence of Case C. The increase of sound pressure levels at high frequencies is very smooth.

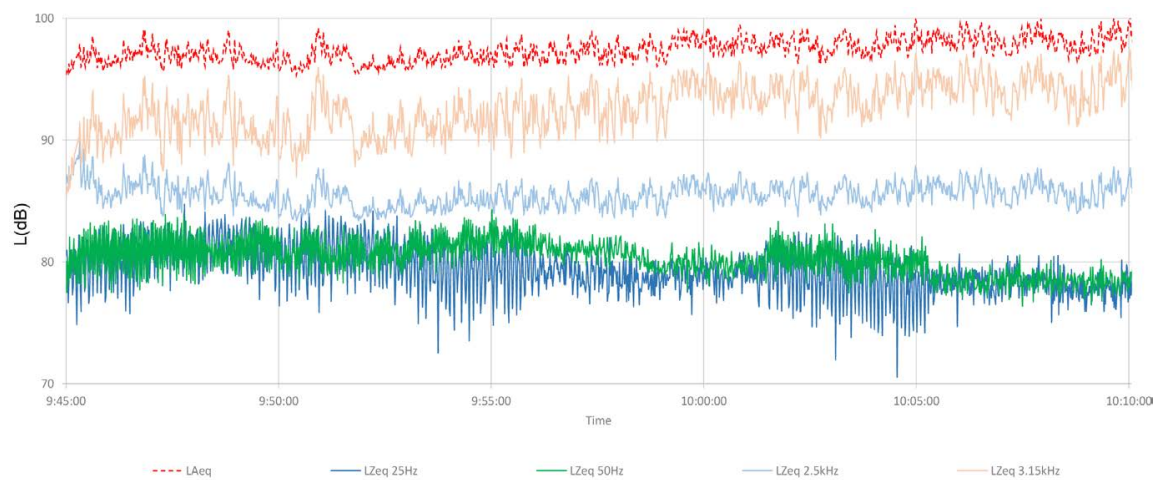

Figure 17. Measured sound pressure levels in selected TOB at the beginning of the episode. Detail of Case C presented in Figure 16. 


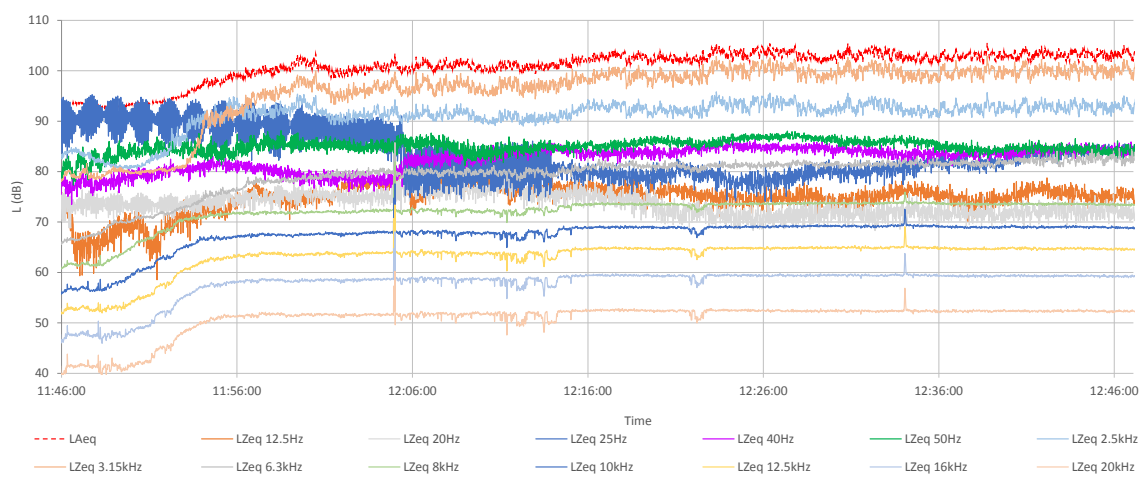

Figure 18. Examples of occurrence of Case C. Full event partially shown in Figure 5 and Figure 13.

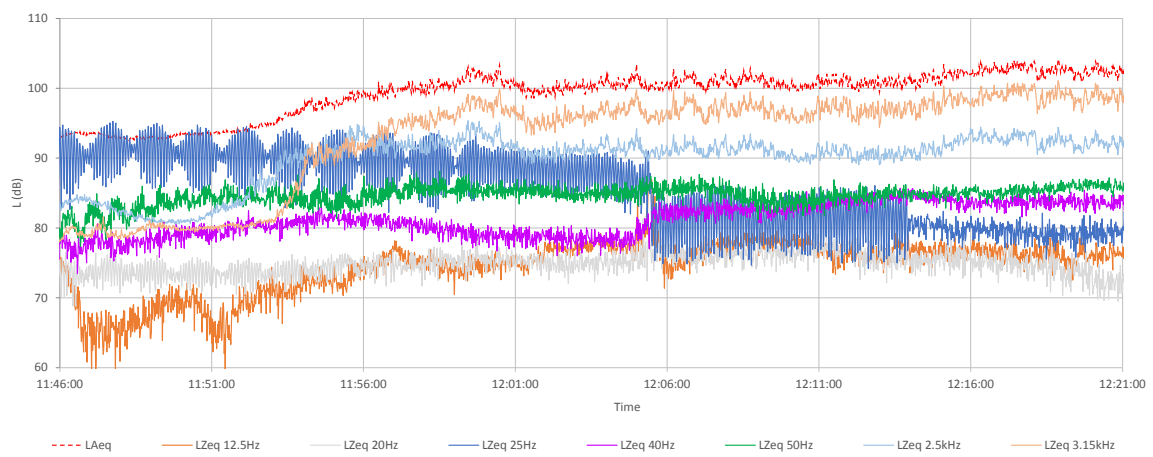

Figure 19. Measured sound pressure levels in selected TOB at the beginning of the event presented in Figure 18.

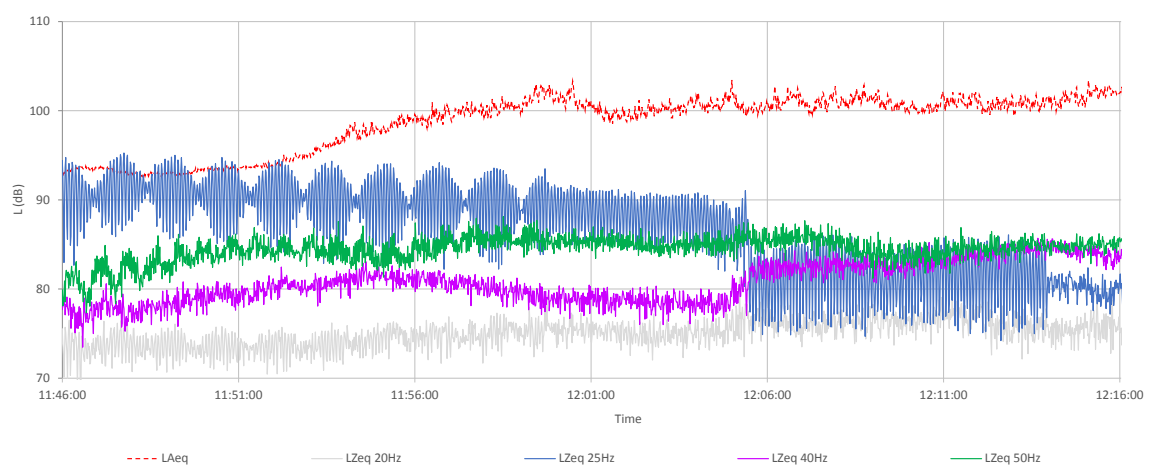

Figure 20. Measured sound pressure levels in selected TOB: $20 \mathrm{~Hz}, 25 \mathrm{~Hz}, 40 \mathrm{~Hz}$ and 50 Hz. Detail of the beginning of the Case C event presented in Figure 18 and Figure 19. Please note that the process of retrieving of chaos lasts more than 25 minutes.

\section{Conclusions}

We have been able to measure the occurrence of several events of thermoacoustic instability in $10 \mathrm{MW}$ engines.

These phenomena occur in large machines in which homogeneity of parameters and simultaneity of processes in the combustion chamber are not proper hypothesis. Small differences and/or fluctuations can generate disturbances and fluctuations in combustion parameters. In these conditions, for example, if a 
coupling occurs with some geometric dimension, the system will go into resonance.

During the preparation of the instability events, processes of loss of chaos in low frequencies, especially in $25 \mathrm{~Hz}$ or $50 \mathrm{~Hz}$, were observed. Also, a reduction of the variability of sound pressure levels in high frequencies occurred. After some minutes, the system retrieves the condition of randomness of the combustion.

Each of the "steady" states of operation lasts several minutes, as so the transition from one to another does.

Every quasi-stationary before and after the occurrence of a thermoacoustic instability episode last a few minutes, so active control systems seem to be a suitable solution to address such problems on large machines.

\section{References}

[1] Strahle, W.C. (1978) Journal of Progress in Energy and Combustion Science, 4, 157-176.

[2] Schemel, C., Thiele, F. and Michel, U. (2004) Numerical Simulation of the Noise Generation at the Outlet Section of Combustion Chambers. CFD-DAGA2004, Strasbourg, Francia.

[3] Litak, G., Taccani, R., Radu, R., Urbanowicz, K., Hołyst, J.A., Wendeker, M. and Giadrossi, A. (2005) Chaos, Solitons and Fractals, 23, 1695-1701.

www.elsevier.com/locate/chaos https://doi.org/10.1016/S0960-0779(04)00434-5

[4] Polifke, W., Paschereit, C.O. and Döbbeling, K. (2001) Journal of Acoustics and Vibration, 6, 135-146. https://doi.org/10.20855/ijav.2001.6.382

[5] Cullick, F.E.C., Lin, W.H., Jahnke, C.C. and Sterling, J.D. (1991) Modeling for Active Control of Combustion and Thermally Driven Oscillations. American Control Conference, 2939-2948, 26-28 June 1991.

http://ieeexplore.ieee.org/stamp/stamp.jsp?tp=\&arnumber $=4791942 \&$ isnumber $=47$ $\underline{91300}$

[6] Ghosh, A. (2008) The Role of Density Gradient in Liquid Rocket Engine Combustion Instability, Chapter 2: Literature Review.

[7] Schuermans, B. (2003) Modeling and Control of Thermoacoustic Instabilities. Ph.D. Thesis, École Polytechnique Fédérale de Lausanne.

[8] Nair, V., Thampi, G., Karuppusamy, S., Gopalan, S. and Sujith, R.I. (2013) International Journal of Spray and Combustion Dynamics, 5, 273-290. https://doi.org/10.1260/1756-8277.5.4.273

[9] Ibrahim, Z.M.A. (2007) An Acoustic Energy Framework for Predicting Combustion-Driven Acoustic Instabilities in Premixed Gas-Turbines. UC San Diego Electronic Theses and Dissertations, Ph.D., UC San Diego. 123 p. Permalink. https://escholarship.org/uc/item/6wn0k48j

[10] Lee, J.G. and Santavicca, D.A. (2005) Experimental Diagnostics of Combustion Instabilities. Chapter 16. In: Lieuwen, T.C. and Yang, V., Eds., Combustion Instabilities in Gas Turbine Engines: Operational Experience, Fundamental Mechanisms, and Modeling, Volume 210, Progress in Astronautics and Aeronautics, American Institute of Aeronautics and Astronautics, Inc. (Frank K. Lu, Editor-in-Chief). Copyright c 2005 by the American Institute of Aeronautics and Astronautics. 
[11] Kabiraj, L. and Sujith, R.I. (2012) Journal of Fluid Mechanics, 713, 376-397

[12] Rogers, D.E. and Marble, F.E. (1956) Jet Propulsion, 1, 456-462.

[13] Asociación Española de Normalización y Certificación - Comité Europeo de Normalización. Norma UNE-EN-ISO 3744:2010. Acústica: Determinación de los niveles de potencia acústica y de los niveles de energía acústica de fuentes de ruido utilizando presión acústica. Métodos de ingeniería para un campo esencialmente libre sobre un plano reflectante (ISO 3744:2010). 84 p. Julio, 2011.

Submit or recommend next manuscript to SCIRP and we will provide best service for you:

Accepting pre-submission inquiries through Email, Facebook, LinkedIn, Twitter, etc. A wide selection of journals (inclusive of 9 subjects, more than 200 journals)

Providing 24-hour high-quality service

User-friendly online submission system

Fair and swift peer-review system

Efficient typesetting and proofreading procedure

Display of the result of downloads and visits, as well as the number of cited articles Maximum dissemination of your research work

Submit your manuscript at: http://papersubmission.scirp.org/

Or contact jmp@scirp.org 